\title{
INTERPOLATION BY COMPLEX SPLINES BY
}

\section{J. TZIMBALARIO}

\begin{abstract}
In this paper we solve the problem of interpolation by certain class of cardinal complex splines. This solution is used to complete the study of cardinal trigonometric splines started in [10] and also to give shorter proofs and to complete the results found for the interpolation problem by complex splines over the unit circle by I. J. Schoenberg [9], J. H. Ahlberg, E. N. Nilson and J. L. Walsh [1].
\end{abstract}

1. Introduction. The problem of complex spline interpolation was initiated by Ahlberg, Nilson and Walsh in a sequence of papers which appeared several years ago, [1], [2] and [3]. This problem was solved by Schoenberg in [9] and almost at the same time by the initiators of the problem. The solutions are completely different and quite complicated. A related problem, the trigonometric spline interpolation,was studied earlier by Schoenberg in a very nice paper, [7]. He connected the study to certain differential operators $\Delta_{m}=D\left(D^{2}+1^{2}\right) \cdots\left(D^{2}+m^{2}\right)(D \equiv d / d n)$. Several generalizations of these topics appeared in literature. Only recently Micchelli [5] returned to Schoenberg's idea by introducing the cardinal $\mathcal{L}$-splines, i.e. splines related to the differential operator $\mathcal{L}=\prod_{j=0}^{n}\left(D-\gamma_{j}\right)$ with $\gamma_{j}$ real numbers. Micchelli gave a complete and systematic treatment to the interpolation problem by cardinal $\mathfrak{L}$-splines, treatment which in our opinion is the most natural one. Schoenberg [6] asked whether Micchelli's theory can be extended to operators $\mathcal{L}$ with imaginary $\gamma$-s. Sharma and the author [10] began such a study for the operator $\Delta_{m}$. Among results connected to other problems, they proved that cardinal trigonometric interpolation at the knots is not unique. This result is only the beginning of the study of such interpolation problems.

The object of this paper is to solve the interpolation problem for cardinal splines related to the operator $\mathcal{L}=\prod_{j=0}^{n}(D-i(l+j) \eta)$ for some $\eta>0$ and $l$ real. This study will put the problem of cardinal trigonometric spline interpolation and complex spline interpolation over the unit circle with equidistant knots in their natural setting. We give complete solution to both problems in a relatively simple way. Moreover, the interpolation points are taken to be not only the knots or half way between the knots, but also any

Received by the editors March 4, 1977.

AMS (MOS) subject classifications (1970). Primary 41A05, 41A15.

Key words and phrases. Cardinal complex splines, Euler-Frobenius polynomials. 
shift of the system of knots. The limiting case of $\eta \rightarrow 0$ is the case of the cardinal polynomial splines so well presented by Schoenberg in his monography [8].

In $\$ 2$ we give all necessary notations and state the interpolation problem. $\$ 3$ deals with an eigenvalue problem closely connected to the main question. Certain auxiliary class of polynomials which is an extension of the EulerFrobenius polynomials is studied in full details. In $\$ 4$ we complete the preparation of all tools needed in the solution of the interpolation problem and we give the main theorem. Finally in $\$ 5$, we treat briefly some applications.

2. Definitions, notations and statement of the main problem. Let $p_{n+1}(x)=$ $\Pi_{j=0}^{n}(x-i(l+j) \eta)$ for some $\eta>0$ and $l$ real. The nullspace of the associated differential operator $\mathcal{L}_{n+1}(D) \equiv p_{n+1}(D)$ is denoted by $\Pi_{n}$.

$$
\Pi_{n}=\left\{y \mid \mathscr{L}_{n+1}(D) y=0\right\} \text {. }
$$

Every element in $\Pi_{n}$ is a linear combination $\sum_{j=0}^{n} c_{j} e^{i(j+l) \eta x}$. The space of cardinal complex splines is defined to be

$$
\delta_{n}=\left\{S(x)\left|S(x) \in C^{n-1}(R), S\right|_{(v h,(v+1) h)} \in \Pi_{n}, \nu \in Z\right\}
$$

for some fix positive $h$.

Complex splines over the unit circle studied by various authors are, in fact, periodic elements of $\delta_{n}$. The cardinal trigonometric splines are real parts of elements in certain $\delta_{n}$. We shall study the following interpolation problem:

$$
S((\nu+\alpha) \eta)=y_{\nu}, \quad \nu \in Z, S(x) \in \delta_{n},
$$

where $y=\left\{y_{\nu}\right\}$ is a given set of complex or real data and $0 \leqslant \alpha<1$.

It can easily be seen that the set of solutions to this problem form a linear manifold in $\delta_{n}$, of dimension $n-1$ if $\alpha=0$ and of dimension $n$ if $0<\alpha<$ 1. Hence the problem requires some boundary conditions and the natural type of conditions are the growth conditions. Let $\gamma \geqslant 0$ and define

$$
\begin{aligned}
Y_{\gamma} & =\left\{\left\{y_{\nu}\right\} \mid y_{\nu}=O\left(|\nu|^{\gamma}\right) \text { as } \nu \rightarrow \pm \infty\right\}, \\
S_{n, \gamma} & =\left\{S(x) \mid S(x) \in \delta_{n}, S(x)=O\left(|x|^{\gamma}\right) \text { as }|x| \rightarrow \infty\right\} .
\end{aligned}
$$

Our main problem is to study existence and uniqueness of elements in $S_{n, \gamma}$ which interpolate sets of data in $Y_{\gamma}$. The class $\delta_{n}^{0}$ of nullsplines for this problem is the class of functions in $\delta_{n}$ which interpolates the zero data, i.e.

$$
\delta_{n}^{0}=\left\{S(x) \mid S(x) \in \delta_{n}, S((\nu+\alpha) h)=0, \nu \in Z\right\} .
$$

The dimension of $\delta_{n}^{0}$ is $n-1$ for $\alpha=0$ and $n$ for $0<\alpha<1$. The study of the uniqueness is based on finding a good basis in $\delta_{n}^{0}$. All discussion depends heavily on locating the zeros of some generalization of Euler-Frobenius polynomials. 
3. The eigenvalue problem. In this section we shall construct solutions to the functional equation

$$
S(x+h)=\lambda S(x), \quad x \in R, S(x) \in S_{n} .
$$

Functions satisfying (3.1) and $S(\alpha h)=0$ belong to $\delta_{n}^{0}$. We shall see later on that this can happen for certain values of $\lambda$ only. The corresponding solutions of (3.1) will be the required basis for $\delta_{n}^{0}$. Let us return to (3.1). The restriction of the solutions to $[0, h]$ satisfies the following set of equations

$$
\begin{gathered}
y^{(j)}(h)=\lambda y^{(j)}(0), \quad j=0,1, \ldots, n-1, \\
y^{(n)}(h)=\lambda y^{(n)}(0)+1, \\
y \in \Pi_{n} .
\end{gathered}
$$

It is easy to see that if $\lambda \notin\left\{e^{i \eta \eta h}, e^{i(l+1) \eta h}, \ldots, e^{i(l+n) \eta h}\right\}$, then the system (3.2) has a unique solution. Let us denote this solution by $A_{n, l}(x, \lambda)$. We set $D_{j} f=(D-i j \eta) f$ for $j$ real and

$$
f^{[j]}=D_{l} D_{l+1} \cdots D_{l+j-1} f \quad\left(f^{[0]}=f\right) .
$$

Following similar lines as Micchelli [5] we can derive some of the properties of $A_{n, l}(x, \lambda)$ formulated in the next theorem.

THEOREM 1.

$$
\begin{gathered}
D_{l} A_{n, l}(x, \lambda)=A_{n-1, l+1}(x, \lambda), \quad D_{l+n} A_{n, l}(x, \lambda)=A_{n-1, l}(x, \lambda), \\
A_{n, l}(x+h)=A_{n, l}(x)+\varphi_{n, l}(x),
\end{gathered}
$$

where $\varphi_{n, l}(x)$ is the unique function in $\Pi_{n}$ which satisfies $\varphi_{n}^{())}(0)=\delta_{j n}, j=$ $0,1, \ldots, n$.

$$
\begin{aligned}
& A_{n, l}^{[j]}(h)=A_{n, l}^{[j]}(0)+\delta_{j, n}, \quad j=0,1, \ldots, n, \\
& \frac{e^{x z}}{e^{z h}-\lambda}=\sum_{n=0}^{\infty} A_{n, l}(x, \lambda) \prod_{k=0}^{n-1}(z-i(l+k) \eta)
\end{aligned}
$$

$$
A_{n, l}(x, \lambda)=\frac{1}{i^{n} n ! \eta^{n}} \sum_{j=0}^{n} \frac{e^{i(l+j) \eta(x-h)}}{1-e^{-i(l+j) \eta h}}(-1)^{n-j}\left(\begin{array}{l}
n \\
j
\end{array}\right) .
$$

REMARK 1 . One can check by direct calculations that the function $\varphi_{n, l}(x)$ is given by

$$
\varphi_{n, l}(x)=e^{i \eta n x} \frac{\left(e^{i \eta x}-1\right)^{n}}{i^{n} n ! \eta^{n}} .
$$

Proof. The proof of (1), (2), (3) and (4) is exactly like that of Theorem 2.1 
in [5]. The Newton expansion (4) is a formal expansion. The coefficients $A_{n, l}(x, \lambda)$ of the expansion are divided differences of the function $e^{x z} /\left(e^{z h}-\right.$ $\lambda)$ at the points $i \eta, i(l+1) \eta, \ldots, i(l+n) \eta$. Using the standard notation for divided differences we have

$$
A_{n, l}(x, \lambda)=\left[i l \eta, \ldots, i(l+n) \eta ; e^{x z} /\left(e^{z h}-\lambda\right)\right] .
$$

Hence

$$
\begin{aligned}
A_{n, l}(x, \lambda) & =\sum_{j=0}^{n} \frac{e^{i(l+j) \eta x}}{e^{i(l+j) \eta h}-\lambda} \cdot \frac{1}{\Pi_{k \neq j}(i j \eta-i k \eta)} \\
& =\frac{1}{i^{n} \eta^{n}} \sum_{j=0}^{n} \frac{e^{i(l+j) \eta x}}{e^{i(l+j) \eta h}-\lambda} \cdot \frac{(-1)^{n-j}}{j !(n-j) !},
\end{aligned}
$$

which is clearly equivalent to (5).

An immediate consequence of (5) in Theorem 1 is the next corollary.

Corollary 1.

$$
A_{n, l}(x, \lambda)=\frac{\Pi_{n, l}(\lambda, x)}{\Pi_{j=0}^{n}\left(e^{i(l+j) \eta h}-\lambda\right)},
$$

where $\Pi_{n, l}(\lambda, x)$ is a polynomial of degree at most $n$ in $\lambda$. Moreover, we can write explicitly the first and the last coefficient of $\Pi_{n, l}(\lambda, x)$.

$$
\Pi_{n, l}(\lambda, x)=(-1)^{n} \varphi_{n, l}(x) \lambda^{n}+\cdots+e^{i(l+n / 2)(n+1) \eta h} \varphi_{n, l}(x-h) .
$$

This shows that if $x=0$, the degree of $\Pi_{n, l}(\lambda, x)$ is at most $n-1$.

The polynomial $\Pi_{n, l}(\lambda, x)$ is a generalization of the Euler-Frobenius polynomials studied by various authors. We arrived at this polynomial by studying restrictions of $(3.1)$ to $(0, h)$. The additional requirement $S(\alpha h)=0$ implies that $\Pi_{n, l}(\lambda, \alpha h)=0$. Hence, we have to find for what values of $\lambda$, $\Pi_{n, l}(\lambda, \alpha h)=0$. At this point we have to use a different approach than that of Micchelli which was based on a theorem by Gantmacher and Krein on oscillatory matrices. The difference is that in our case we deal with complex polynomials and the other approach was real. We will derive a recursion formula for $\Pi_{n, l}(\lambda, x)$, which ultimately, will help solve the main problem.

Theorem 2. (1) The polynomial $\Pi_{n, l}(\lambda, x)$ defined by (3.6) satisfies the following recursion formula

$$
\Pi_{n, l}(\lambda, x)=\frac{\left(e^{i l \eta h}-\lambda\right)}{i n \eta} \Pi_{n-1, l+1}(\lambda, x)-\frac{\left(e^{i(l+n) \eta h}-\lambda\right)}{i n \eta} \Pi_{n-1, l}(\lambda, x) .
$$

(2) The $\lambda$-zeros of $\Pi_{n, l}(\lambda, x)$ are all simple and located on the half line $-\rho e^{i(l+n / 2) \eta h}, \rho \geqslant 0$, provided $\eta h<\underline{2 \pi /(n+1) \text { and } 0}<x<h$. 
(3) $\Pi_{n, l}(\lambda, x)=\lambda^{n} e^{-i(l+n / 2)(n+1) \eta h} \Pi_{n, l}\left(\bar{\lambda}^{-1}, h-x\right)$.

REMARK 2. If $\eta \rightarrow 0$ we obtain for $x=0$ and $h / 2$ the two variants of Euler-Frobenius polynomials discussed by Schoenberg [8]. In this case all zeros are negative.

REMARK 3. If $x=0$, by part (2) of Theorem 1 and part (3) of Theorem 2, we find that if $\lambda$ is a zero of $\Pi_{n, l}(\lambda, 0)$, then $\bar{\lambda}^{-1}$ is also a zero, i.e. $\Pi_{n, l}(\lambda, 0)$ is a reciprocal polynomial. If $x=h / 2$, we see directly from part (3) of Theorem 2 that $\Pi_{n}(\lambda, h / 2)$ is again a reciprocal polynomial. Hence $\Pi_{n, l}\left(-e^{i(l+n / 2) \eta h}, 0\right)=0$ only if $n$ is even and $\Pi_{n, l}\left(-e^{i(l+n / 2) \eta h}, h / 2\right)=0$ only if $n$ is odd.

In the proof of part (2) of this theorem, we require the following elementary lemma concerning polynomials with real coefficients. This result was proved in [1], but does not appear there in an explicit form.

LEMMA 1. Let $p_{n}(z)=\Pi_{j=1}^{n}\left(z-\lambda_{j}\right)$ with $\lambda_{n}<\lambda_{n-1}<\cdots<\lambda_{1}<0$. Denote by $\xi_{1}, \ldots, \xi_{n-1}$ the critical points of $p_{n}(z)$, i.e. the zeros of $p_{n}^{\prime}(z)$. Then the locus of $\operatorname{Im} p_{n}(z)=0$ is the real axis together with curves perpendicular to the real axis at the critical points $\xi_{j}(j=1, \ldots, n-1)$, symmetric with respect to the real axis and asymptotic to the directions $k \pi / n$ and $(2 n-j) \pi / n$, $j=1, \ldots, n-1$. Along the level curve $\operatorname{Im} p_{n}(z)=0$ through $\xi_{j}(j=1, \ldots, n$ $-1), \operatorname{Re} p_{n}(z)$ has the same sign as $(-1)^{j}$, respectively.

Proof of Theorem 2. Since $A_{n, l}(x, \lambda)$ is a certain divided difference of $e^{x z} /\left(e^{z h}-\lambda\right)$, we can write immediately, on using elementary properties of divided differences, that

$$
A_{n, l}(x, \lambda)=\frac{A_{n-1, l+1}(x, \lambda)-A_{n-1, l}(x, \lambda)}{i n \eta} .
$$

Substituting (3.6) in (3.9) we get (3.8).

The following proof of part (2) is similar to the proof of Theorem 2 in [1] with some necessary modifications. From the definitions of $\Pi_{n, l}(\lambda, x)$ and $A_{n, l}(x, \lambda)$ we find that for arbitrary real $\mu$

$$
\Pi_{n, l}(\lambda, x)=e^{i \mu \eta(x+(n+1) h)} \Pi_{n, l-\mu}\left(\lambda e^{-i \mu \eta h}, x\right) .
$$

Substituting (3.10) in (3.9) with $\mu=l+n / 2$, changing the variable $\lambda=$ $\rho e^{i(l+n / 2) \eta h}$ and denoting $\tilde{\Pi}_{n}(\rho, x)=\Pi_{n,-n / 2}(\rho, x)$, we get after some calculations

$$
\begin{aligned}
\tilde{\Pi}_{n}(\rho, x)= & \frac{e^{i \eta x / 2}-\rho e^{i m \eta h / 2}}{i \eta \eta} \tilde{\Pi}_{n}\left(\rho e^{-i \eta h / 2}, x\right) \\
& -\frac{e^{-i \eta x / 2}-\rho e^{-i m \eta h / 2}}{i n \eta} \tilde{\Pi}_{n}\left(\rho e^{i \eta h / 2}, x\right) .
\end{aligned}
$$


By a simple induction we see that $\tilde{\Pi}_{n}(\rho, x)$ is a polynomial with real coefficients. In order to complete the proof of part (2) is enough to show that $\tilde{\Pi}_{n}(\rho, x)$ has only simple, real, negative roots. We proceed by induction. For $n=1$,

$$
\tilde{\Pi}_{1}(\rho, x)=\frac{2}{\eta}\left(-\rho \sin \frac{\eta h}{2}+\sin \frac{\eta(x-h)}{2}\right) .
$$

Let us assume that the zeros of $\Pi_{n-1}(\rho, x)$ are $\lambda_{n-1}<\lambda_{n-2}<\cdots<\lambda_{1}<0$. Multiplying (3.11) by $\rho$, we get

$$
\begin{aligned}
\rho \tilde{\Pi}_{n}(\rho, x) & =\frac{1}{i \eta \eta}\left(e^{i \eta(x+h) / 2}-\rho e^{i \eta(x+h) / 2}\right) \rho e^{-i \eta h / 2} \tilde{\Pi}_{n-1}\left(\rho e^{-i \eta h / 2}, x\right) \\
& -\frac{1}{i \eta \eta}\left(e^{-i \eta(x+h) / 2}-\rho e^{-i \eta(x+h) / 2}\right) \rho e^{i \eta h / 2} \tilde{\Pi}_{n-1}\left(\rho e^{i \eta h / 2}, x\right) .
\end{aligned}
$$

By the induction assumption, the polynomial $\rho \tilde{\Pi}_{n-1}(\rho, x)$ satisfies the conditions of Lemma 1. Let us denote his critical points by $\xi_{n-1}<\xi_{n-2}$ $<\cdots<\xi_{1}<0$. Construct two lines through 0 inclined at $\pm \eta h / 2$ to the positive axis. The level curve $\operatorname{Im}\left\{z \tilde{\Pi}_{n-1}(z, x)\right\}=0$ through $\xi_{j}(j=1, \ldots, n$ -1) will intersect the above lines at the points $x_{j} e^{-i m h / 2}, x_{j} e^{i m h / 2}$ (the existence of these points is insured by the conditions of the theorem). By Lemma 1 and by (3.7) we have

$$
\operatorname{sgn} \operatorname{Re} \tilde{\Pi}_{n-1}\left(x_{j} e^{-i \eta h / 2}, x\right)=(-1)^{n-1+j} .
$$

Rewriting (3.12), we get

$$
\begin{aligned}
\rho \tilde{\Pi}_{n}(\rho, x)= & \frac{2}{n \eta}\left(\sin \frac{\eta(x+h)}{2}-\rho \sin \frac{(n+1) \eta h}{2}\right) \\
& \cdot \operatorname{Re}\left\{\rho e^{\left.-i \eta h / 2 \tilde{\Pi}_{n-1}\left(\rho e^{-i \eta h / 2}, x\right)\right\}}\right. \\
& +\frac{2}{m \eta}\left(\cos \frac{\eta(x+h)}{2}-\rho \cos \frac{(n+1) \eta h}{2}\right) \\
& \cdot \operatorname{Im}\left\{\rho e^{i \eta h / 2} \tilde{\Pi}_{n-1}\left(\rho e^{i \eta h / 2}, x\right)\right\} .
\end{aligned}
$$

Since $\sin [\eta(x+h) / 2]$ and $\sin [(n+1) \eta h / 2]$ are both positive and $\rho<0$, we get

$$
\operatorname{sgn} \tilde{\Pi}_{n}\left(x_{j}, x\right)=(-1)^{n+j}, \quad j=1, \ldots, n-1 .
$$

By (3.7) we have also $\operatorname{sgn} \tilde{\Pi}_{n}(0-, x)=(-1)^{n}$ and $\operatorname{sgn} \tilde{\Pi}_{n}(-\infty, x)=1$. Hence $\tilde{\Pi}_{n}(\rho, x)$ must have $n$ negative simple roots. Let us proceed to the proof of part (3). By part (5) of Theorem 1, we have 


$$
\begin{aligned}
A_{n, l}(h & \left.-x, \bar{\lambda}^{-1}\right)=\frac{1}{i^{n} n ! \eta^{n}} \sum_{j=0}^{n} \frac{e^{-i(l+j) \eta(h-x)}}{e^{i(l+j) \eta h}-\bar{\lambda}^{-1}}(-1)^{n-j}\left(\begin{array}{l}
n \\
j
\end{array}\right) \\
& =-\frac{\bar{\lambda}}{i^{n} n ! \eta^{n}} \sum_{j=0}^{n} \frac{e^{-i(l+j) \eta x}}{e^{-i(l+j) h}-\bar{\lambda}}(-1)^{n-j}\left(\begin{array}{c}
n \\
j
\end{array}\right)=(-1)^{n-1} \bar{\lambda} \overline{A_{n, l}(x, \lambda)} .
\end{aligned}
$$

On using the definition of $\Pi_{n, l}(\lambda, x)$ we complete the proof.

4. The interpolation problem. We mentioned in $\$ 2$, that the solution to the interpolation problem depends on finding a proper basis for $\mathfrak{S}_{n}^{0}$.

LEMMA 2. Let $\lambda_{j}=-\rho_{j} e^{i(l+n / 2) \eta h}, j=1, \ldots, n, \rho_{j}>0$ be the roots of the polynomial $\Pi_{n, l}(\lambda, \alpha h), 0<\alpha<1$. Define $S_{j}(x), x \in R, j=1, \ldots, n$, as

$$
S_{j}(x)=A_{n, l}(x, \lambda) \text { for } 0<x<h
$$

and

$$
S_{j}(x+h)=\lambda_{j} S_{j}(x), \quad x \in R .
$$

Then $S_{j}(x), j=1, \ldots, n$, form a basis for $\delta_{n}^{0}$. If $\alpha=0$, we have a similar statement with $n$ replaced by $n-1$.

Proof. Since $\lambda_{j}$ are distinct, the functions $S_{j}(x)$ exhibit different types of exponential growth, so they are linearly independent. Since their number is equal to the dimension of $\mathfrak{S}_{n}^{0}$, they span $\mathfrak{S}_{n}^{0}$.

Now we shall study how many values of $\alpha$ can give $\Pi_{n, l}(\lambda, \alpha h)=0$ for a certain value of $\lambda$. This is done by finding an additional recursion formula.

THEOREM 3. (1)

$$
\begin{aligned}
\frac{\partial}{\partial x} \tilde{\Pi}_{n}(\rho, x)= & \frac{1}{2}\left(e^{i \eta x / 2}-\rho e^{i \eta n h / 2}\right) \tilde{\Pi}_{n-1}\left(\rho e^{-i \eta h / 2}, x\right) \\
& +\frac{1}{2}\left(e^{-i \eta x / 2}-\rho e^{-i \eta n h / 2}\right) \tilde{\Pi}_{n-1}\left(\rho e^{i \eta h / 2}, x\right) .
\end{aligned}
$$

(2) If $\rho>0$ and $\rho \notin\left\{e^{i m h / 2}, e^{i(n / 2+1) \eta h}, \ldots, e^{i m \eta h / 2}\right\}$, then $\tilde{\Pi}_{n}(\rho, x) \neq 0$ on $(0, h)$.

(3) If $\rho<0$ and $\eta h<2 \pi /(n+1)$, then $\tilde{\Pi}_{n}(\rho, x)$ has exactly one simple $x$-zero in $[0, h)$.

Proof. Part (2) is an immediate consequence of the fact that $\tilde{\Pi}_{n}(\rho, x)$ has only real negative zeros in $\rho$ for $x \in[0, h)$. Let us proceed to the proof of part (1). By Theorem 1, part (1), we have

$$
D_{n / 2} A_{n,-n / 2}(x, \rho)=A_{n-1,-n / 2}(x, \rho) .
$$

Hence, by the definition of $\Pi_{n,-n / 2}(\rho, x)$ we have

$$
\left(D-i \frac{n}{2} \eta\right) \frac{\Pi_{n,-n / 2}(\rho, x)}{\Pi_{j=0}^{n}\left(e^{i(-n / 2+j) \eta h}-\rho\right)}=\frac{\Pi_{n-1,-n / 2}(\rho, x)}{\Pi_{j=0}^{n-1}\left(e^{i(-n / 2+j) \eta h}-\rho\right)}
$$


where $D=\partial / \partial x$. By (4.4) and the definition of $\tilde{\Pi}_{n}(\rho, x)$, we get

$D \tilde{\Pi}_{n}(\rho, x)=\frac{i n \eta}{2} \tilde{\Pi}_{n}(\rho, x)+\left(e^{i \eta \eta h / 2}-\rho\right) e^{i \eta(x+n h) / 2} \tilde{\Pi}_{n-1}\left(\rho e^{i \eta h / 2}, x\right)$.

Substituting (3.8) in (4.5) we get part (1).

For the proof of part (3) we use some continuity arguments. If $\eta \rightarrow 0$, the situation reduces to the special case of cardinal polynomial spline. The result is known [8], [10] in this case, but also can be proved directly by a simple induction. It is easy to see that $\tilde{\Pi}_{n}(\rho, x)$ is continuous as a function of the parameter $\eta$. Assuming that part (3) is not true in general, there is some value of $\eta \neq 0$ and some value of $\rho<0$ for which $\tilde{\Pi}_{n}(\rho, x)=0$ and $\partial \tilde{\Pi}_{n}(\rho, x) / \partial x$ $=0$ for some $x$ in $[0, h)$. Substituting these values of $\eta, \rho$ and $x$ in part (1) of Theorem 3 and in (3.11) we get

$$
\left(e^{i \eta x / 2}-\rho e^{i \eta \eta h / 2}\right) \tilde{\Pi}_{n-1}\left(\rho e^{-i \eta h / 2}, x\right)=0 .
$$

Since $\eta h<2 \pi /(n+1)$ and $x \in[0, h), e^{i \eta x / 2} \neq \rho e^{-i \eta \eta h / 2}$ and hence, $\tilde{\Pi}_{n-1}\left(\rho e^{-i \eta h / 2}, x\right)=0$. This is in contradiction to the fact that all $\lambda$ zeros of $\tilde{\Pi}_{n-1}(\rho, x)$ are real and negative. Now we can state the main theorem.

THEOREM 4. Let $0<\alpha<1$ and $\eta h \leqslant 2 \pi /(n+1)$. For any set of data $\left\{y_{v}\right\}$ in $Y_{\gamma}$, there is a unique spline in $\delta_{n, \gamma}$ satisfying

$$
S((\nu+\alpha) h)=y_{\nu}, \quad \nu \in Z,
$$

if and only if for $n$ odd $\alpha \neq \frac{1}{2}$ and for $n$ even $\alpha \neq 0$.

REMARK 4. It is worth noting that for $\alpha \neq 0, \frac{1}{2}$ the solution exists and is unique for any $n$.

REMARK 5. The case $\eta \rightarrow 0$ coincides with the case $n=1$ in [4].

Once we proved Theorems 2 and 3, the process of proving Theorem 4 is already a standard process. This is well presented in [5] and [8], so we will omit here the proof.

\section{Applications.}

A. The cardinal trigonometric interpolation. Let $n=2 m$ and $l=-m$. In this case the polynomials $A_{n, l}(, \lambda)$ and $\Pi_{n, l}(\lambda, x)$ are polynomials with real coefficients. Moreover, $A_{n, l}(x, \lambda)$ coincides with the polynomial $\operatorname{Am}(x, t)$ connected to the problem of cardinal trigonometric interpolation. Hence following the same lines as the proof of Theorem 4, we get a result much stronger than Theorem 3 in [10]. More precisely, we can prove that if $0<\alpha<1$ and if the data is of power growth, the interpolation problem of finding $S(x) \in \delta\left(\Delta_{m}, \eta\right)$ (see the notations of [10]) such that $S((\nu+\alpha) \eta)=$ $y_{\nu}$ and $S(x)$ is of power growth, admits a unique solution if and only if $\alpha \neq 0$.

B. The spline interpolation of periodic sequences. Let $\left\{y_{\nu}\right\}$ be a periodic sequence of period $k>2$. Since $\left\{y_{v}\right\}$ is bounded, under the conditions of 
Theorem 4, i.e. $\eta<2 \pi /(n+1)$, if $n$ odd $\alpha \neq \frac{1}{2}$ and if $n$ even $\alpha \neq 0$, there is a unique bounded interpolant $S(x)$ in $\delta_{n}$. It is easy to see that $S(x)$ must be also periodic of period $k$ [8]. Denote by $\delta_{n, k}$ the class of elements in $\delta_{n}$ which are $k$ periodic. If $n \geqslant k-1, S_{n, k}$ reduces to the class of complex polynomials of degree $n$ in $e^{i \eta x}$ multiplied by $e^{i l n x}$ [9]. The nontrivial cases occur if $n<k-1$.

We are interested to know what happens in the case $n$ odd, $\alpha=\frac{1}{2}$ and $n$ even $\alpha=0$. In $S_{n, k}$ we can have solutions of $S(x+h)=\lambda S(x)$ if and only if $\lambda=\omega^{j}, j=0,1, \ldots, k-1$, where $\omega=e^{2 \pi i / k}$. Denote by $S_{j}(x), j=$ $0,1, \ldots, k-1$, to be the elements in $\delta_{n, k}$ satisfying $S(x+h)=\omega^{j} S(x)$. Each of these splines is unique up to a constant factor. It can be verified easily that the functions $S_{j}(x)$ are linearly independent. Moreover, they span $\delta_{n, k}$. Any element $S(x)$ in $\delta_{n, k}$ can be written as

$$
S(x)=\sum_{j=0}^{k-1} c_{j} S_{j}(x) \text {. }
$$

Clearly we get existence and uniqueness for the interpolation problem if and only if the linear system

$$
S((\nu+\alpha) h)=\sum_{j=0}^{k-1} c_{j} S_{j}((\nu+\alpha) h)=0, \quad \nu=0,1, \ldots, k-1,
$$

has only the trivial solution $c_{j}=0, j=0,1, \ldots, k-1$. Since $S_{j}(x+h)=$ $\omega^{j} S_{j}(x)$, we get from (5.2)

$$
\sum_{j=0}^{k-1} c_{j} \omega^{\nu j} S_{j}(\alpha h)=0, \quad \nu=0,1, \ldots, k-1 .
$$

Hence $c_{j} S_{j}(\alpha h)=0$, for $j=0,1, \ldots, k-1$, and then, is necessary and sufficient for the existence and uniqueness that $S_{j}(\alpha h) \neq 0, j=0,1, \ldots, k$ -1 . In other words, if and oniy if none of the $\omega^{j}, j=0,1, \ldots, k-1$, is a zero of $\Pi_{n, l}(\lambda, \alpha h)$. Since we deal only with the exceptional cases, this polynomial is reciprocal. By Theorem 2, part (2) and Corollary $1, \omega^{j} \neq$ $-e^{i(l+n / 2) \eta h}$ for all $j=0,1, \ldots, k-1$. In the special case $h=1, l=0$, $\eta=2 \pi / k$, the above condition reduces to $j \neq(n+k) / 2$, for all $j=$ $0,1, \ldots, k-1$. This is the case of complex polynomial spline over the unit circle studied in [1] and [9]. In conclusion we proved that there is a unique solution to the interpolation problem except for the cases: (i) $\alpha=0, n$ and $k$ even numbers, (ii) $\alpha=\frac{1}{2}, n$ and $k$ odd numbers. We point out that the cases $\alpha \neq 0, \frac{1}{2}$ was not studied in the previous treatment.

\section{REFERENCES}

1. J. H. Ahlberg, E. N. Nilson and J. L. Walsh, Complex polynomial splines on the unit circle, J. Math. Anal. Appl. 33 (1971), 234-257. 
2. __ Complex cubic splines, Trans. Amer. Math. Soc. 129 (1967), 391-413.

3. __ Properties of analytic splines, J. Math. Anal. Appl. 27 (1969), 262-278.

4. S. L. Lee, A. Sharma and J. Tzimbalario, A class of cardinal splines with Hermite type interpolation, J. Approximation Theory 18 (1976), 30-38.

5. C. A. Micchelli, Cardinal L-splines, Splines and Approximation Theory, Academic Press, New York, 1976.

6. I. J. Schoenberg, On Charles Micchelli's theory of cardinal L-splines, Splines and Approximation Theory, Academic Press, New York, 1976.

7. ___ On trigonometric spline interpolation, J. Math. Mech. 13 (1964), 795-826.

8. Cardinal spline interpolation, CBMS Regional Conf. Monograph 12, SIAM, Philadelphia, 1973.

9. On polynomial spline functions on the unit circle. I, II, Proc. Conf. Theoretic Functions, Akademiai Kiado, Budapest, 1971.

10. A. Sharma and J. Tzimbalario, A class of cardinal trigonometric splines, SIAM J. Math. Anal. 7 (1970), 809-819.

Department of Mathematics, Ben Gurion University of the Negev, BeER SheVa 84 120, ISRAEL

Current address: 2/215 Hertzel Street, Rehovot, Israel 